\title{
Prevalence and drug resistance profile of Mycobacterium tuberculosis isolated from pulmonary tuberculosis patients attending two public hospitals in East Gojjam zone, northwest Ethiopia
}

Kelemework Adane ${ }^{1,2,3^{*}}$, Gobena Ameni², Shiferaw Bekele ${ }^{3}$, Markos Abebe ${ }^{3}$ and Abraham Aseffa ${ }^{3}$

\begin{abstract}
Background: The spread of multidrug-resistant tuberculosis (MDR-TB) strains has become a challenge to the global TB control and prevention program. In Ethiopia, particularly in rural areas, information on drug-resistant TB is very limited. In this study, we determined the drug resistance patterns of Mycobacterium tuberculosis (M. tuberculosis) isolates from pulmonary TB patients attending two public hospitals in the East Gojjam zone of northwest Ethiopia.

Methods: A cross-sectional study was conducted between May 2011 and January 2012 using Region of difference-9 (RD9) typing for the identification of species mycobacterium. Drug susceptibility testing (DST) of M. tuberculosis isolates to the first-line drugs: isoniazid, rifampicin, ethambutol and streptomycin was performed by the indirect proportion method on Middle brook 7H10 Agar media.

Results: Out of 385 pulmonary TB suspects studied, 124 (32.2\%) were culture positive among which 120 were M. tuberculosis strains. Susceptibility testing was performed for 89 isolates. Resistance to at least one drug was $15.58 \%$ ([12/77], 95 \% Cl: 7.48-23.68) among newly diagnosed and $50.0 \%$ ([6/12], 95 \% Cl: 21.71-78.29) among previously treated cases. Resistance among newly diagnosed patients was most common for streptomycin $5.19 \%(4 / 77)$ and ethambutol $5.19 \%$ (4/77) followed by rifampicin $3.89 \%$ (3/77). Among retreatment cases, isoniazid resistance was most frequent in which $33.33 \%(4 / 12)$ of the isolates were resistant. MDR prevalence was $1.29 \%(1 / 77)$ for newly diagnosed and $16.67 \%(2 / 12)$ for retreatment cases. In a multivariate logistic regression analysis, age group of 25-34 years (adjusted $\mathrm{OR}=4.24 ; 95 \% \mathrm{Cl}: 1.02-17.5 ; \mathrm{P}=0.046$ ) and previous history of treatment (adjusted $\mathrm{OR}=5.42 ; 95 \% \mathrm{Cl}$ : 1.56-27.49; $\mathrm{P}=0.01$ ) were independently associated with anti-TB drug resistance.

Conclusions: In general, the magnitude of anti-TB drug resistance including MDR-TB was comparable to previous studies in other areas of Ethiopia. However, rifampicin resistance was high, which could suggest the potential for a rise in the incidence of MDR. Therefore, re-enforcing TB control programs should be considered by the concerned public health authorities.
\end{abstract}

Keywords: Mycobacterium tuberculosis, Drug resistance, MDR-TB, Ethiopia

\footnotetext{
* Correspondence: ingoldmlt@gmail.com

${ }^{1}$ Institute of Biomedical Sciences, College of Health Sciences, Mekelle

University, PO Box 1872, Mekelle, Ethiopia

${ }^{2}$ Aklilu Lemma Institute of Pathobiology, Addis Ababa University, PO Box

1176, Addis Ababa, Ethiopia

Full list of author information is available at the end of the article
} 


\section{Background}

The emergence of drug-resistant tuberculosis is a critical threat to tuberculosis (TB) control and is a major public health concern in several countries. Multidrug-resistant TB (MDR-TB), defined as resistant to at least isoniazid and rifampicin, is emerging as a major clinical and public health challenge in areas of sub-Saharan Africa [1]. Ethiopia is one of the high TB and HIV burdened countries in sub-Saharan Africa which ranks 7th among the 22 high TB burden countries in the world [1]. According to the WHO 2013 report, the prevalence and incidence rates of TB in the country were 224/100,000 and 247/ 100,000 populations, respectively. The rate of MDR-TB was estimated to be $1.6 \%$ and $12 \%$ of new and retreatment cases, respectively [1].

In countries with high burden of $\mathrm{TB}$, continuous surveillance and regular monitoring of drug resistance based on routine drug susceptibility testing (DST) of TB patients is essential to assess the magnitude and trends of anti-TB drug resistance [1, 2]. However, in Ethiopia, there is limited capacity to perform culture and DST of M. tuberculosis, even from patients suspected of harbouring drug-resistant strains. As a consequence, many of MDR-TB patients remain undiagnosed which negatively impacts the global strategy of halting and eliminating TB [3].

Few studies previously conducted in Ethiopia indicated drug resistance proportions ranging from 14.6\%-30.1\% [4-6] and $11.1 \%-42.1 \%[4,5]$ among new and previously treated patients, respectively. However, most studies were conducted in urban areas and might not be representative for rural remote regions of the country. Besides, in Ethiopia, the socioeconomic, lifestyle and environmental conditions differ across regions, varying from agricultural based to pastoral communities and from semi-arid to humid fertile areas [7], and could affect the distribution of drug-resistant $M$. tuberculosis strains [8]. The current study was conducted among patients attending health facilities in the East Gojjam zone of the Amhara region in northwest Ethiopia. East Gojjam zone is one of the rural areas where the communities lead their life mainly by farming and to date there is no report on anti-tuberculosis drug resistance from this part of the country. This study therefore determined the prevalence of drug-resistant and MDR-TB among pulmonary TB patients attending two public hospitals in the region from May 2011 to January 2012.

\section{Methods}

\section{Study setting}

This cross-sectional study was conducted in Debre Markos Referral and Mota District hospitals located in the East Gojjam zone of the Amhara region, northwest Ethiopia. The prevalence of all forms of TB and smear-positive TB in the Amhara Regional State was reported to be 643 and 168 per 100,000 populations, respectively [9]. East Gojjam zone is divided into 18 administrative districts and agriculture is the major source of income for more than $90 \%$ of its population. There are two public hospitals, more than 18 health centers, about 384 health posts, and 5 higher and 25 medium private clinics providing service for about 3.5 million people in their catchment areas. In addition to the two hospitals, all the 18 health centers provide sputum smear microscopy and directly observed treatment short course (DOTS) service. Moreover, the 5 higher and most medium private clinics have currently implemented the DOTS service $[10,11]$. We conducted the current study at the two public hospitals due to the fact that they represent the largest center for TB diagnosis (referral sites for rural communities) and considering feasibility.

\section{Study population}

From May 2011 to January 2012, a total of 385 pulmonary TB suspected patients presenting at the two hospitals were consecutively enrolled. Both smear-positive and smear-negative patients were included. Inclusion criteria were: age $\geq 15$ years, willingness to participate in the study (with informed consent including for HIV testing) and clinical suspicion of pulmonary TB strong enough to warrant a direct sputum smear for acid-fast bacilli (AFB). Prior to sputum collection, data on demographic information and history of previous treatment were collected using a structured questionnaire. Then, three consecutive sputum samples (spot, morning and spot) were collected from each patient. Direct microscopic examination of sputum for AFB after Ziehl- Neelsen staining was performed at the laboratories of the respective hospitals. Then samples were stored at $4{ }^{\circ} \mathrm{C}$ for a maximum of 5 days until transported in a cold box to the Armauer Hansen Research Institute (AHRI) in Addis Ababa for culturing.

\section{HIV testing}

Testing for HIV was done according to the current National algorithm recommended by the Federal Ministry of Health of Ethiopia. Two rapid HIV tests, HIV $(1+2)$ Rapid Test Strip (KHB) and Stat-Pak were run sequentially. Samples were tested first with KHB. Positive samples were confirmed with Stat-Pak. Discordant results were resolved using a third confirmatory test kit, HIV-1/2 Unigold Recombinant assay. Pre and post-test HIV counseling was provided for all consenting individuals.

\section{Mycobacterial Culture}

Sputum specimens were processed by the Petroff's method following the standard operational procedures. In brief, an equal volume of sputum specimen was mixed with an equal volume of $4 \% \mathrm{NaOH}$ and concentrated at $3000 \mathrm{rpm}$ 
for $15 \mathrm{~min}$. The sediment was neutralized with $2 \mathrm{~N} \mathrm{HCL}$, using phenol red as indicator, to prepare the inoculums for culture. Then, three Lowenstein-Jensen slants, two containing $0.75 \%$ glycerol and one containing $0.6 \%$ pyruvate, were inoculated per each sputum sample. Culture tubes were incubated at $37{ }^{\circ} \mathrm{C}$ and inspected for growth of colonies weekly for up to 8 weeks. Mycobacteria were confirmed with acid-fast bacilli (AFB) staining.

\section{Region of difference 9 (RD9) deletion typing for identification of $M$. tuberculosis}

DNA was extracted by heat killing of the mycobacterial colonies. Briefly, heat killed bacterial suspensions were obtained by heating cultures suspended in Tris-EDTA (TE) at $80{ }^{\circ} \mathrm{C}$ for one hour followed by incubation in an ultrasonic bath for $5 \mathrm{~min}$. Region of Difference analysis was performed by using RD9 FlankFW, RD9 Int and RDFlankRev primers as described previously [12]. Interpretation of results was made on the basis of detection of bands of different sizes [13]. For quality control, $M$. tuberculosis $\mathrm{H} 37 \mathrm{Rv}$ and M. bovis BCG (as positive control) and water (as a non-template control) were included.

\section{Conventional drug susceptibility testing}

Drug susceptibility testing (DST) was performed by the indirect proportion method on the enriched Middlebrook 7H10 agar media using a 24 well plate as recommended by Bert van Klingeren et al. [14]. Stock solutions of each drug were prepared using the appropriate solvent and the following final drug concentrations (critical concentrations) were used: isoniazid (INH), $0.2 \mu \mathrm{g} / \mathrm{ml}$, rifampicin (RIF), $1.0 \mu \mathrm{g} / \mathrm{ml}$, ethambutol (EMB), $5.0 \mu \mathrm{g} / \mathrm{ml}$ and streptomycin (STM), $2.0 \mu \mathrm{g} / \mathrm{ml}$ as recommended elsewhere [15]. Standardized bacterial suspension for each isolate was distributed in $10 \mu \mathrm{l}$ volumes into 9 wells of the 24-well plate containing 7H10 medium with a precise concentration of the selected anti-TB drugs and into the 10th well containing drug free medium. The 11th well of each plate was inoculated with a mycobacterial solution containing $1 \%$ of the suspension (1:100 suspensions). This was followed by incubation at $35^{\circ} \mathrm{C}$ in the presence of sufficient humidity and bacterial growth was checked on the 12th, 19th and 28th days. If the growth on drug containing media with 1:1 bacterial suspensions was greater than the growth on drug free control with 1:100 bacterial suspensions, the isolate was defined as resistant and if it was lower, the isolate was defined as susceptible to that particular drug. The test was repeated if the growth was equal or nearly equal for both. For quality control, the reference strain of M. tuberculosis H37Rv (ATCC 27294), which is susceptible to the entire standard anti tuberculosis drugs, was used in each batch of media prepared with drugs.
Resistance to a particular drug with or without resistance to any of the other anti-TB drugs was denoted as 'any resistance'. An MDR-TB patient was defined as one whose sputum isolates showed resistance to isoniazid and rifampicin with or without resistance to other antiTB drugs. Poly-resistance was defined as resistance to more than one drug with the exception of the combination of isoniazid and rifampicin.

\section{Data analysis}

Data were entered using Epi Data entry version 3.1 software and analyzed using SPSS version 21. Differences between sputum culture positive and culture negative groups were investigated using the $x^{2}$ test (categorical variables). Age was categorized into four levels. Bivariate and multivariate logistic regression analysis was performed to examine the association of independent variables with resistance outcome. Covariates with p-values of $\leq 0.25$ in the bivariate analysis were included in the multivariate model. HIV status was considered as a potential confounder and hence was assessed. Accordingly, the multivariate model consisted of age (age group of 15-24 years as a referent), treatment category (new cases as referent) and HIV status (HIV-negative patients as a referent). Comparisons between subgroups (new cases vs. retreatment cases, HIV-positive vs. HIVnegative, and age groups) with resistance outcome were expressed as odds ratios (OR) with a $95 \%$ confidence interval (CI). P-values of $\leq 0.05$ were considered as statistically significant.

\section{Ethical issues}

Ethical clearance was obtained from Aklilu Lemma Institute of Pathobiology, Addis Ababa University (AAU) and AHRI/ALERT research ethics review committees. Written informed consent was obtained from the study participants and assent from parents/guardians for those under 18 years of age. For illiterate participants, data collectors informed each respondent and confirmed the willingness of participants by signing on the informed consent sheet. Moreover, confidentiality was assured for all information provided. Confirmed MDR cases were reported to the study hospitals for treatment.

\section{Results}

\section{Characteristics of the study population}

Of 385 pulmonary TB (PTB) suspects enrolled, 244 (63.4 \%) were males and 141 (36.6\%) females with an age range of 15 to 73 and mean age of 36.8 years. The majority of the study participants, 326 (84.7\%), were rural residents and more than three-fourths, 295 (76.6 \%) were farmers. Illiterates numbered 267 (69.4 \%) and 118 (30.6\%) had completed primary school or above. Ninety-two $(23.9 \%)$ of the study participants 
reported close contact with a TB patient and mostly $(89.0 \%)$ at home. Eighty-nine (23.1 \%) were HIV positive, 79 (20.5\%) were smear-positive, and 68 (17.7 \%) had a previous history of anti-TB therapy.

\section{Culture result and relation to patient characteristics} Of 385 sputum samples, 124 (32.2\%) (from 82 male and 42 female patients) were culture positive. Eight ( $2.1 \%)$ were contaminated. Seventy-seven $(97.5 \%)$ of the smear-positive and $47(18.1 \%)$ of the smear-negative sputum samples were culture positive. Two (2.6 \%) smear-positive specimens were culture negative. Thirtyfour $(36.9 \%)$ of the 92 participants who reported close contact with a TB patient were culture positive. Positive cultures were more frequent among HIV-negative than HIV-positive participants, but the difference was not statistically significant $(P=0.093)$. There was no significant difference between the culture-positive and the culturenegative groups in terms of HIV status, age distribution, sex, and history of previous TB treatment (Table 1).

\section{Region of difference (RD) based species typing}

Region of difference based multiplex PCR for RD9 (RD9 analysis) showed that out of 124 isolates, 120 were $M$. tuberculosis species. The remaining 4 isolates failed to show any band on RD9 typing. No M. bovis was identified in this study.

\section{Drug susceptibility patterns}

Phenotypic drug susceptibility testing results were available for 89 (74 \%) of the 120 isolates. However, the sociodemographic and clinical characteristics of patients whose isolates were not available for drug susceptibility testing were not significantly different from those included except that the loss was relatively higher in smear-negative culture positive cases.

Resistance to at least one drug was detected in 18 (20.23\%, 95 \% CI: 11.86-28.54) of the isolates of which 12 (20.7 \%) were from males and 6 (19.4\%) from females. The highest proportions of any drug resistance were observed in STM 7 (7.87 \%, 95 \% CI: 2.3-13.5) followed by INH 6 (6.74 \%, $95 \%$ CI: 1.51-11.89) and EMB 6 (6.74 \%, $95 \%$ CI: 1.51-11.89). Three isolates (3.37 \%, $95 \%$ CI: 0.0-7.17) were MDR and 2 (2.24 \%) were poly resistant. One MDR isolate was resistant to all the four anti-TB drugs tested. A summary of drug susceptibility pattern of the isolates is shown in Table 2.

Anti-TB drug resistance was more frequent among retreatment patients than newly diagnosed cases (50.0\% vs.15.58 \%) and this difference was statistically significant (crude OR $=5.42 ; 95 \% \mathrm{CI}: 1.49-19.64, \mathrm{P}=0.01$ ). The isolate which was resistant to the four anti-TB drugs tested was also isolated from a previously treated patient.

The HIV prevalence among the study population for whom drug resistance testing result available $(n=89)$ was $19.1 \%(17 / 89)$. The occurrence of drug resistance was not related to HIV infection status (crude OR = 2.72; $95 \%$ CI: $0.85-8.88$; $\mathrm{P}=0.093$ ), to positivity by microscopy of sputum smears (crude OR $=1.08 ; 95 \% \mathrm{CI}$ : $0.36-3.24 ; \mathrm{P}=0.88$ ) or gender (crude $\mathrm{OR}=1.52 ; 95 \% \mathrm{CI}$ : $0.48-4.69 ; \mathrm{P}=0.48$ ). However, we found a significant association between age and anti-TB drug resistance; those in the group of 25-34 years were more likely to have drug-resistant TB (crude OR $=4.67$; $95 \%$ CI: 1.25-17.44; $\mathrm{P}=0.022$ ) as compared to patients in other age groups. All the three MDR strains were also isolated from rural HIV-seronegative patients in the age group of 25-34. After fitting into the multivariate logistic regression model, age (adjusted $\mathrm{OR}=4.24 ; 95 \% \mathrm{CI}: 1.02-17.5 ; \mathrm{P}=0.046$ ) and

Table 1 Baseline characteristics of participants enrolled in the study and their association with culture outcome as analyzed by the $\mathrm{x} 2$ test

\begin{tabular}{llll}
\hline Characteristic & Participants with positive sputum culture $(\mathrm{n}=124), \mathrm{n}(\%)$ & Participants with negative sputum culture $(\mathrm{n}=261), \mathrm{n}(\%)$ & $P$ value \\
\hline Sex & $82(66.1)$ & $162(62.0)$ \\
Male & $42(33.9)$ & $99(38.0)$ \\
Female & $102(82.3)$ & $67(25.7)$ \\
HIV status & $194(74.3)$ \\
HIV-negative & $22(17.7)$ & \\
HIV-positive & $107(86.3)$ & $51(19.5)$ \\
Previous history of treatment & $210(80.5)$ \\
No & $17(13.7)$ & 256 \\
Yes & $77(62.1)$ & $2(0.7)$ \\
Sputum microscopy & $47(37.7)$ & $259(99.3)$ \\
Smear-positive & & 0.093 \\
Smear-negative &
\end{tabular}

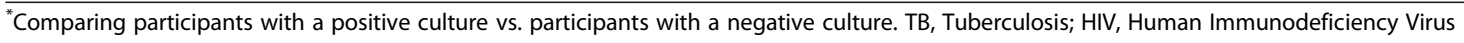


Table 2 Drug resistance patterns of M.tuberculosis strains isolated from newly diagnosed and previously treated pulmonary TB patients attending two public hospitals in East Gojjam zone, northwest Ethiopia

\begin{tabular}{|c|c|c|c|c|}
\hline Drug resistance pattern & Isolates from new cases, n (\%) & $\begin{array}{l}\text { Isolates from previously } \\
\text { treated cases, n (\%) }\end{array}$ & Total, n (\%) & $95 \% \mathrm{Cl}$ \\
\hline & $77(86.5)$ & $12(13.5)$ & $89(100.0)$ & \\
\hline \multicolumn{5}{|l|}{ Any resistance } \\
\hline Resistance to any drug & $12(15.58)$ & $6(50.0)$ & $18(20.23)$ & $11.86-28.54$ \\
\hline Any INH & $2(2.59)$ & $4(33.33)$ & $6(6.74)$ & $1.51-11.89$ \\
\hline Any RIF & $3(3.89)$ & $2(16.67)$ & $5(5.62)$ & $0.82-10.38$ \\
\hline Any EMB & $4(5.19)$ & $2(16.67)$ & $6(6.74)$ & $1.51-11.89$ \\
\hline Any STM & $4(5.19)$ & $3(25.0)$ & $7(7.87)$ & $2.3-13.52$ \\
\hline \multicolumn{5}{|l|}{ Mono resistance } \\
\hline INH alone & 0 & $1(8.3)$ & $1(1.12)$ & $0.0-3.27$ \\
\hline RIF alone & $2(2.59)$ & 0 & $2(2.24)$ & $0.0-5.25$ \\
\hline EMB alone & $4(5.19)$ & $1(8.33)$ & $5(5.62)$ & $0.82-10.38$ \\
\hline STM alone & $3(3.89)$ & 0 & $3(3.37)$ & $0.0-7.14$ \\
\hline \multicolumn{5}{|c|}{ Resistance to more than one drug } \\
\hline INH + RIF only (MDR) & $1(1.29)$ & $1(8.33)$ & $2(2.24)$ & $0.0-5.25$ \\
\hline $\mathrm{INH}+\mathrm{RIF}+\mathrm{EMB}+\mathrm{STM}$ only & 0 & $1(8.33)$ & $1(1.12)$ & $0.0-3.27$ \\
\hline INH + STM only & 0 & $2(16.67)$ & $2(2.24)$ & $0.0-5.25$ \\
\hline
\end{tabular}

$\mathrm{Cl}$, confidence interval; TB, tuberculosis; INH, isoniazid; STM, streptomycin; RIF, rifampicin; EMB, ethambutol

previous history of treatment (adjusted OR $=5.42 ; 95 \% \mathrm{CI}$ : 1.56-27.49; $\mathrm{P}=0.01$ ) remained independently associated with occurrence of anti-TB drug resistance (Table 3).

\section{Discussion}

In this study, about a quarter of M.tuberculosis isolates were resistant to at least one of the anti-TB drugs tested (20.23\%). This is higher than a previous report in Gondar (6.7 \%) [16] but comparable to a resistance proportion reported in Addis Ababa (22.3 \%) [4]. However, a relatively higher resistance proportion (60.8\%) has been reported in another study from Addis Ababa [17]. The possible explanation for this difference could be due to the fact that this study was conducted at St Peter's TB specialized hospital in Addis Ababa which is a referral site for complicated TB cases from all parts of the country. When compared to studies from other African countries, our figure is also higher than a report from South Africa (7.3 \%) [18], but lower than a Kenyan study report (30.1\%) [19].

The most frequent resistance proportion was observed in STM (7.87 \%). This drug has been in use since the beginning of TB chemotherapy and hence a higher proportion of STM resistance is expected. However, our figure is lower than previous studies in Ethiopia which reported $26.6 \%$ [20] and $19.0 \%$ [4] of STM resistance. The relatively lower STM resistance in our study could be attributed to time differences in that STM is no more a first line drug (for most cases at least), since the intensive phase was replaced with oral drugs which could lead improved proportions of sensitive isolates with time in recent years. The decreasing proportion of STM resistance could be an advantage as the drug is in the regimen for the treatment of TB patients in category II and for other bacterial infections.

Resistance to INH in our study was $6.74 \%$ which is slightly higher than an earlier report in Addis Ababa (5.5\%) [20] but lower than other reports in Ethiopia in which $17 \%$ [4] and 29.4\% [21] of the isolates were INH resistant. Compared to recent studies in other regions of Africa, our figure is comparable to a report in Rwanda (6.2\%) [22], higher than from Uganda (3.2 \%) [22, 23], but lower than a finding in Kenya (12.9\%) [19]. Previous studies conducted in 2002 and 2006 in Addis Ababa reported that $4.1 \%$ [5] and $2.3 \%$ [17] of the isolates were INH mono resistant in new cases and zero in previously treated cases $[5,17]$. In our study, INH mono-resistance was detected only in 1 (1.1\%) of the isolates from a previously treated case.

The proportion of EMB resistance in this study (6.74\%) is higher than previous findings in Ethiopia which ranged from $0.0 \%$ to $5.2 \%[4,24,25]$. Monoresistance to EMB was also higher in new cases (5.19\%). This implies the existence of ongoing transmission of drug-resistant strains in the study sites and could indicate weakness in TB prevention and control measures as it is accompanied by mono-resistance to RIF in new cases as well. Since EMB enhances the effect of many other drugs including beta- 
Table 3 Bivariate and multivariate analysis for patient related risk factors for any drug resistance among pulmonary TB patients attending two public hospitals in East Gojjam zone, northwest Ethiopia ( $n=89$ )

\begin{tabular}{|c|c|c|c|c|c|c|}
\hline \multirow[t]{2}{*}{ Characteristic } & \multicolumn{2}{|c|}{ Any drug resistance } & \multirow[t]{2}{*}{ COR $(95 \% \mathrm{Cl})$} & \multirow[t]{2}{*}{ P-value } & \multirow[t]{2}{*}{ AOR $(95 \% \mathrm{Cl})$} & \multirow[t]{2}{*}{$p$-value } \\
\hline & Yes, n (\%) & No, n (\%) & & & & \\
\hline \multicolumn{7}{|l|}{ Sex } \\
\hline Male & $13(72.2)$ & $45(63.4)$ & $1.52(0.48-4.69)$ & 0.48 & & \\
\hline Female & $5(27.8)$ & $26(36.6)$ & & & & \\
\hline \multicolumn{7}{|l|}{ HIV-status } \\
\hline HIV-positive & $6(33.3)$ & $11(15.5)$ & $2.72(0.85-8.81)$ & 0.093 & $2.76(0.72-10.54)$ & 0.14 \\
\hline HIV-negative & $12(66.7)$ & $60(84.5)$ & & & & \\
\hline \multicolumn{7}{|c|}{ Age group (in years) } \\
\hline $15-24$ & $4(22.2)$ & $28(39.4)$ & & & & \\
\hline $25-34$ & $10(55.6)$ & $15(21.1)$ & $4.67(1.25-17.44)$ & 0.022 & $4.24(1.02-17.5)$ & 0.046 \\
\hline $35-44$ & $3(16.6)$ & $17(23.9)$ & $1.24(0.25-6.21)$ & 0.79 & $0.99(0.18-5.65)$ & 0.99 \\
\hline$\geq 45$ & $1(5.6)$ & $11(15.6)$ & $0.64(0.064-6.35)$ & 0.71 & $0.59(0.05-6.36)$ & 0.66 \\
\hline \multicolumn{7}{|c|}{ Previous history of treatment } \\
\hline Yes & $6(33.3)$ & $6(8.4)$ & $5.42(1.49-19.64)$ & 0.01 & $6.55(1.56-27.49)$ & 0.01 \\
\hline No & $12(66.7)$ & 65 (91.6) & & & & \\
\hline \multicolumn{7}{|c|}{ Smear microscopy } \\
\hline Smear-positive & $12(66.7)$ & $46(64.8)$ & $1.087(0.36-3.24)$ & 0.88 & & \\
\hline Smear-negative & $6(33.3)$ & $25(35.2)$ & & & & \\
\hline
\end{tabular}

AOR, Adjusted Odds Ratio; HIV, Human Immunodeficiency Virus; Cl, Confidence Interval, COR, Crude Odds Ratio

lactam drugs on different mycobacterial species, the rise of EMB resistance affects the advantage that could be exploited to develop a regimen for the management of MDR-TB [26].

Although the lowest proportion of resistance observed in this study was in RIF (5.62 \%), this is higher than previous reports in Ethiopia (0.1 \% to $1.4 \%$ ) [5, 24, 25]. Monoresistance to RIF was observed in $2.59 \%$ of the isolates which is also higher than previous reports $[5,24]$. The relatively higher proportion of resistance to this drug in this study might be attributed to the fact that RIF is largely used in Ethiopia for the treatment of other bacterial infections. Since RIF is the most important drug to treat TB, emergence of resistance to this drug has huge implications for TB control policies. Even if the small sample size might limit us to generalize, our findings suggest that the precursors of MDR-TB are increasing in the study areas as RIF resistance predicts MDR [26]. This could hamper the effectiveness of the new RIF based six-month treatment regimen adopted by the Federal Ministry of Health of Ethiopia. Hence, proper use of this drug should be ensured in the study sites.

In this study, two factors were found to be associated with anti-TB drug resistance; age group of 25-34 years and having had a previous history of TB treatment. Several studies documented the association between a previous history of TB treatment and anti-TB drug resistance $[4,25,26]$. With regard to age group, it is difficult to directly compare with previous reports as different studies used different cut-off points for age groups. A study in South Africa, for instance, showed that age groups of 35-54 and $>54$ years were strongly associated with anti-TB drug resistance [27]. The association observed in our study cloud be due to the high-risk behavior of young people and hence a tendency to interrupt TB treatment.

Multi drug resistance (MDR) was observed in 3 (3.37\%) isolates in which $2(16.67 \%)$ were from previously treated patients and one (1.29\%) from a newly diagnosed case. Previous studies reported MDR proportions ranging from $0.6 \%$ to $2.7 \%[4,17,24,28]$ and $3.5 \%$ to $15.7 \%[5,21,29]$ among new and previously treated patients, respectively. Our result of MDR-TB among newly diagnosed patients is higher than a report in Addis Ababa (0.6\%), a study in major towns of Amhara region (1.0\%) [24] and the WHO estimate of $0.1 \%$ primary MDR-TB in Ethiopia [1]. This indicates that there is a progressive increase of MDR over years imposing a serious public health threat. However, it is also worthy to mention that the current magnitude of anti-TB drug resistance in the study sites might have changed since the isolates were collected nearly four years ago. We recommend further large scale studies to be conducted in the underlying population to quantify the problem more precisely.

Although few studies have reported an association between HIV infection and development of drug resistance 
[30], our study did not find any association between HIV infection status and anti-TB drug resistance. This lack of association between HIV infection status and mycobacterial drug resistance is consistent with the results of earlier studies in Addis Ababa [4,5] and studies from other sub-Saharan African countries [31, 32]. In our study, the lack of association could be due to the low number of known HIV positive cases included in the study.

Our study was not without pitfalls. First, as the study was conducted among TB patients seeking treatment only at the two hospitals in the zone (health centers and private clinics not included), data may not be representative of TB patients in the population of East Gojjam zone. Moreover, the fact that our sample size was small might have influenced our estimation of anti-TB drug resistance proportion in the study sites. However, the current study provides important data regarding drug resistance in rural hospital settings and could be used by authorities in the region to initiate appropriate interventions.

\section{Conclusions}

Overall, anti-TB drug resistance including MDR-TB is comparable to previous studies in other parts of Ethiopian. However, the relatively higher rate of RIF resistance observed in our study signals the danger of increasing MDRTB in the study areas in the future. On the other hand, the higher level of MDR-TB among previously treated patients suggests the need to strengthen the DOTS strategy and the capacity of laboratories to undertake DST especially among previously treated patients.

\section{Abbreviations}

AHRI/ALERT: Armauer Hansen Research Institute/All African Leprosy, Tuberculosis and Rehabilitation Training Center; DOTS: Directly observed treatment short course; DST: Drug susceptibility testing; EMB: Ethambutol; HCL: Hydrochoric acid; HIV: Human Immunodeficiency Virus; INH: Isoniazid; $\mathrm{NaOH}$ : Sodium hydroxide; MDR: Multidrug-resistance; OR: Odds ratio; RD: Region of difference; RIF: Rifampicin; STM: Streptomycin; TB: Tuberculosis; WHO: World Health Organization.

\section{Competing interests}

The authors declare that there is no any competing interest.

\section{Authors' contributions}

KA was involved in the study conception and design, culturing and drug susceptibility testing, data analysis and drafting of the manuscript. AA and GA were involved in the design and reviewing of the manuscript. SB was involved in culturing and drug susceptibility testing while MA was involved in reviewing of the manuscript. All authors have read and approved the final version of the manuscript.

\section{Acknowledgments}

The authors are grateful to laboratory professionals of Debre Markos and Mota hospitals, particularly to Mr Derejew Zewdie and Shimeles Yizengaw for their invaluable contribution during data collection. We would also like to thank Armauer Hasen Research Institute (AHRI) and Addis Ababa University for their financial and material support.

\section{Author details}

Institute of Biomedical Sciences, College of Health Sciences, Mekelle University, PO Box 1872, Mekelle, Ethiopia. ${ }^{2}$ Aklilu Lemma Institute of Pathobiology, Addis Ababa University, PO Box 1176, Addis Ababa, Ethiopia.

${ }^{3}$ Armauer Hansen Research Institute, PO Box 1005, Addis Ababa, Ethiopia.

Received: 2 September 2014 Accepted: 12 June 2015

Published online: 20 June 2015

\section{References}

1. World Health Organization. Global tuberculosis report. 2013.

2. World Health Organization. Anti-tuberculosis drug resistance in the world: Prevalence and trends. 2000

3. Federal Ministry of Health of Ethiopia. Manual for National Tuberculosis and Leprosy Control Programme. Ethiopia: Ministry of Health, Addis Ababa; 2008.

4. Eyob G, Guebrexabher H, Lemma E, Wolday D, Gebeyehu M, Abate G, et al. Drug susceptibility of Mycobacterium tuberculosis in HIV-infected and-uninfected Ethiopians and its impact on outcome after 24 months of follow-up. Int J Tuberc Lung Dis. 2004;8(11):1388-91.

5. Bruchfeld J, Aderaye G, Palme IB, Bjorvatn B, Ghebremichael S, Hoffner S, et al. Molecular epidemiology and drug resistance of Mycobacterium tuberculosis isolates from Ethiopian pulmonary tuberculosis patients with and without human immunodeficiency virus infection. J J Clin Microbiol. 2002;40(5):1636-43.

6. Yimer S, Bjune G, Alene G. Diagnostic and treatment delay among pulmonary tuberculosis patients in Ethiopia: a cross sectional study. BMC Infect Dis. 2005;5(1):112.

7. Abbink JG. New configurations of Ethiopian ethnicity: the challenge of the South. Northeast Afr Studi. 1998;59-81.

8. Narasimhan P, Wood J, Maclntyre CR, Mathai D. Risk factors for tuberculosis. Pulmonary Medicine. 2013;2013.

9. Amhara National Regional State Health Bureau. Fifth National TB Research Conference. Bahir Dar: ANRS/TBLCP; 2009. p. 1-37.

10. Asemahagn $M$. Assessing the quality of tuberculosis laboratory services in selected public and private health facilities in Western Amhara Ethiopia. J Med Diagn Meth. 2014;3(158):2.

11. Amhara Regional Health Bureau (ARHB). Second quarter health sectors' performance report. Bahir Dar, Ethiopia. 2005.

12. Huard RC, de Oliveira Lazzarini LC, Butler WR, van Soolingen D, Ho JL. PCR-based method to differentiate the subspecies of the Mycobacterium tuberculosis complex on the basis of genomic deletions. J Clin Microbiol. 2003;41(4):1637-50.

13. World Health Organization. Laboratory services in tuberculosis control for the global tuberculosis programme. 1998.

14. Van Klingeren B, Dessens-Kroon M, van der Laan T, Kremer K, van Soolingen D. Drug susceptibility testing of Mycobacterium tuberculosis complex by use of a high-throughput, reproducible, absolute concentration method. J Clin Microbiol. 2007;45(8):2662-8.

15. World Health Organization. Guidelines for surveillance of drug resistance in tuberculosis. 4th ed. 2009.

16. Biadglegne F, Tessema B, Sack U, Rodloff AC. Drug resistance of Mycobacterium tuberculosis isolates from tuberculosis lymphadenitis patients in Ethiopia. IJMR. 2014;140(1):116.

17. Agonafir M, Lemma E, Wolde-Meskel D, Goshu S, Santhanam A, Girmachew $F$, et al. Phenotypic and genotypic analysis of multidrug-resistant tuberculosis in Ethiopia. Int J Tuberc Lung Dis. 2010;14(10):1259-65.

18. Churchyard G, Corbett E, Kleinschmidt I, Mulder D, De Cock K. Drug-resistant tuberculosis in South African gold miners: incidence and associated factors. Int J Tuberc Lung Dis. 2000;4(5):433-40.

19. Ndung'u PW, Kariuki S, Revathi G. Resistance patterns of Mycobacterium tuberculosis isolates from pulmonary tuberculosis patients in Nairobi. Int J Tuberc Lung Dis. 2011;6(01):33-9.

20. Kassu Desta DA, Lemma E, Gebeyehu M, Feleke B. Drug susceptibility of Mycobacterium tuberculosis isolates from smear negative pulmonary tuberculosis patients, Addis Ababa, Ethiopia. Ethiop J Health Dev. 2008;22(2):212-5.

21. Hussein B, Debebe T, Wilder-Smith A, Ameni G. Drug susceptibility test on Mycobacterium tuberculosis isolated from pulmonary tuberculosis patients in three sites of Ethiopia. Afr J Microbiol Res. 2013;7(9):791-6. 
22. Umubyeyi AN, Vandebriel G, Gasana M, Basinga P, Zawadi J, Gatabazi J, et al. Results of a national survey on drug resistance among pulmonary tuberculosis patients in Rwanda. Int J Tuberc Lung Dis. 2007;1 1(2):189-94.

23. Bazira J, Asiimwe BB, Joloba ML, Bwanga F, Matee MI. Mycobacterium tuberculosis spoligotypes and drug susceptibility pattern of isolates from tuberculosis patients in South-Western Uganda. BMC Infect Dis. 2011;11(1):81.

24. Yimer SA, Agonafir M, Derese Y, Sani Y, Bjune GA. HOLM-HANSEN C. Primary drug resistance to anti-tuberculosis drugs in major towns of Amhara region, Ethiopia. Apmis. 2012;120(6):503-9.

25. Demissie M, Gebeyehu M, Berhane Y. Primary resistance to anti-tuberculosis drugs in Addis Ababa Ethiopia. Int J Tuberc Lung Dis. 1997;1(1):64-7.

26. Abate $\mathrm{G}$, Miörner H. Susceptibility of multidrug-resistant strains of Mycobacterium tuberculosis to amoxycillin in combination with clavulanic acid and ethambutol. J Antimicrob Chemother. 1998;42(6):735-40.

27. Green E, Obi C,Nchabeleng M, De Villiers B, Sein P, Letsoalo T, et al. Drug-susceptibility patterns of Mycobacterium tuberculosis in Mpumalanga Provice, South Africa: Possible guiding design of retreatment regimen. J Health Popul Nut. 2010; 28(1):7.

28. Abebe G, Abdissa K, Abdissa A, Apers L, Agonafir M, De-Jong BC, et al. Relatively low primary drug resistant tuberculosis in southwestern Ethiopia. BMC Res Notes. 2012;5(1):225.

29. Mitike $G$, Kebede D, Yeneneh $H$. Prevalence of antituberculosis drug resistance in Harar Tuberculosis Centre Ethiopia. East Afr Med J. 1997;74(3):158-61.

30. Ramaswamy S, Musser J. Molecular genetic basis of antimicrobial agent resistance in Mycobacterium tuberculosis update. Tubercle Lung Dis. 1998:79(1):3-29.

31. Noeske J, Nguenko PN. Impact of resistance to anti-tuberculosis drugs on treatment outcome using World Health Organization standard regimens. Trans R Soc Trop Med Hyg. 2002;96(4):429-33.

32. Murray J, Sonnenberg P, Shearer SC, Godfrey-Faussett P. Human immunodeficiency virus and the outcome of treatment for new and recurrent pulmonary tuberculosis in African patients. Am J Respir Crit Care Med. 1999;159(3):733-40

\section{Submit your next manuscript to BioMed Central and take full advantage of:}

- Convenient online submission

- Thorough peer review

- No space constraints or color figure charges

- Immediate publication on acceptance

- Inclusion in PubMed, CAS, Scopus and Google Scholar

- Research which is freely available for redistribution 